\title{
Perinatal fluoxetine treatment and dams' early life stress history have opposite effects on aggressive behavior while having little impact on sexual behavior of male rat offspring
}

\author{
Danielle J. Houwing ${ }^{1}$ • Diana C. Esquivel-Franco ${ }^{1}$ - Anouschka S. Ramsteijn ${ }^{1} \cdot$ Kirsten Schuttel $^{1}$ • Eline L. Struik ${ }^{1}$. \\ Chantal Arling ${ }^{1}$. Sietse F. de Boer ${ }^{1}$. Jocelien D. A. Olivier ${ }^{1}$
}

Received: 28 October 2019 / Accepted: 22 April 2020 / Published online: 17 July 2020

(C) The Author(s) 2020

\begin{abstract}
Rationale Many depressed women continue antidepressant treatment during pregnancy. Selective serotonin reuptake inhibitor (SSRI) treatment during pregnancy increases the risk for abnormal social development of the child, including increased aggressive or defiant behavior, with unknown effects on sexual behavior.

Objectives Our aim was to investigate the effects of perinatal SSRI treatment and maternal depression, both separately and combined, on aggressive and sexual behavior in male rat offspring.

Methods Heterozygous serotonin transporter (SERT ${ }^{ \pm}$) knockout dams exposed to early life stress (ELSD) were used as an animal model of maternal depression. Early life stress consisted of separating litters from their mother for $6 \mathrm{~h}$ a day on postnatal day (PND)2-15, resulting in a depressive-like phenotype in adulthood. Depressive-like dams were treated with fluoxetine (FLX, $10 \mathrm{mg} / \mathrm{kg}$ ) or vehicle throughout pregnancy and lactation (gestational day 1 until PND 21). Male offspring were tested for aggressive and sexual behavior in adulthood. As lifelong reductions in SERT expression are known to alter behavioral outcome, offspring with normal $\left(\mathrm{SERT}^{+/+}\right)$and reduced $\left(\mathrm{SERT}^{ \pm}\right)$SERT expression were assessed.

Results Perinatal FLX treatment reduced offensive behavior and the number of animals attacking and increased the latency to attack, especially in SERT ${ }^{+/+}$offspring. Perinatal FLX treatment reduced the mounting frequency in $\mathrm{SERT}^{+/+}$offspring. ELSD increased offensive behavior, without affecting sexual behavior in SERT ${ }^{ \pm}$offspring.

Conclusions Overall, our research demonstrates that perinatal FLX treatment and ELSD have opposite effects on aggressive behavior, with little impact on sexual behavior of male offspring.
\end{abstract}

Keywords SSRI $\cdot$ Development $\cdot$ Serotonin transporter $\cdot$ Early life stress $\cdot$ Behavior $\cdot$ Offspring $\cdot$ Depression

\section{Introduction}

Many women who initiate or continue antidepressant treatment during pregnancy are prescribed selective serotonin reuptake inhibitors (SSRIs), as they are considered relatively safe for both mother and child (Gentile 2005). However, SSRIs are able to cross the placenta and are excreted into breast milk, thus reaching the developing child (Heikkinen et al. 2003; Noorlander et al. 2008). SSRIs act on the serotonergic system by blocking the serotonin transporter, resulting in sustained higher extracellular serotonin (5-HT) levels and increased

Jocelien D. A. Olivier

j.d.a.olivier@rug.nl

1 Department of Neurobiology, GELIFES, University of Groningen, Nijenborgh 7, 9747, AG Groningen, the Netherlands serotonergic neurotransmission (Pierz and Thase 2014). In the developing brain, 5-HT acts as a neurotrophic factor, regulating a wide variety of neurodevelopmental processes including neurogenesis, cell division, differentiation and migration, neuroapoptosis, and synaptic plasticity (Azmitia 2001; Gaspar et al. 2003; Sodhi and Sanders-Bush 2003). Therefore, it has been suggested that perinatal SSRI exposure has the potential to influence serotonergic functioning and subsequently alter behavioral development of the child. Indeed, exposure to SSRIs has been linked to abnormal development of social behaviors (reviewed by Gemmel et al. 2018a). Clinical studies show that children from mothers treated with SSRIs during pregnancy have an increased risk to show more externalizing behaviors such as aggression or defiant behavior (Oberlander et al. 2007) and more internalizing behaviors such as anxiety, depression, and social withdrawal (Hanley et al. 2015; Oberlander et al. 2010). Furthermore, there is an ongoing discussion about 
whether SSRI treatment increases the risk for the child to develop autism spectrum disorder, as effects often disappear when controlling for maternal illness (Brown et al. 2017; Kaplan et al. 2017; Zhou et al. 2018). In preclinical studies, it has been shown that developmental SSRI exposure affects various social behaviors in rodents, including aggressive and sexual behavior. For instance, treating rat dams with fluoxetine (FLX) during the prenatal period increased the number of fighting bouts in adult male offspring, without affecting attack latency (Singh et al. 1998). Treating mice dams with FLX during the prenatal and early postnatal period resulted in an increased number of offspring attacking intruder mice, even though they showed a similar amount of aggressive behavior compared with control mice (Kiryanova and Dyck 2014). Concerning sexual behavior, offspring from mice dams treated with FLX from conception until weaning showed reduced sexual incentive motivation, without affecting copulatory behaviors (Gouvêa et al. 2008). In rats, a decrease in copulatory behaviors including number of mounts, intromission, and ejaculations has been found after exposure to the SSRI citalopram during the early postnatal period (Harris et al. 2012; Maciag et al. 2006; Rodriguez-Porcel et al. 2011). These data indicate that perinatal SSRI exposure increases aggressive behavior, while decreasing sexual behavior. Interestingly, similar neural structures are involved in intermale aggression and reproductive behavior (Anderson 2012). Even though the pathways of reproductive and offensive behaviors are shared, exposure to perinatal FLX appears to have opposite effects on aggressive and sexual behavior in male rodent offspring. Since it is difficult to discern the effects of maternal depression and SSRI treatment in humans, animal models of maternal stress are of great help to separate the effects of maternal depression and SSRI treatment during pregnancy on offspring behavior. From a translational perspective, studying the effects of perinatal SSRI exposure in an animal model of depression is highly relevant, as usually depressed and not healthy women take SSRIs. In rodents, stress during the prenatal period has been associated with reduced aggression in male offspring (Kinsley and Svare 1986; Patin et al. 2005). In contrast, stressors during the early postnatal period, such as maternal separation or social deprivation, have been found to increase aggressive behavior in rodents (Sandi and Haller 2015). Besides aggressive behavior, it has also been shown that both prenatal and early postnatal stress in rodents reduce male sexual behavior, especially the number of copulatory behaviors (Masterpasqua et al. 1976; Rhees and Fleming 1981), and result in less animals showing at least one intromission or ejaculation response (Hernández-Arteaga et al. 2016; Rhees et al. 2001). The number of preclinical studies combining the effects of maternal stress and SSRI exposure during the perinatal period to investigate offspring neurodevelopment is increasing but still limited when it comes to aggressive and sexual behavior. Kiryanova et al. (2016) studied the effects of FLX treatment in mouse dams exposed to prenatal chronic unpredictable tress.
They found that perinatal FLX treatment increased levels of aggressive behavior, while prenatal stress reduced levels of aggressive behavior in male offspring mice (Kiryanova et al. 2016). Interestingly, when FLX treatment was administered in dams exposed to early life stress, offspring displayed normal aggressive behavior. When it comes to sexual behavior, Rayen et al. (2013) found that FLX treatment during lactation reduced the number of intromissions and resulted in a longer latency to the first intromission and ejaculation in male offspring. However, prenatal restraint stress and the combination of prenatal stress and FLX treatment did not affect copulatory behavior of male offspring (Rayen et al. 2013). While these studies use an animal model of prenatal stress, pregestational stress might be more clinically relevant, as many women cope with a depression long before they become pregnant. Therefore, we investigated the effects of perinatal FLX treatment on aggressive and sexual behavior in male offspring using an animal model of pregestational maternal depression. Our animal model of maternal depression consists of heterozygous serotonin transporter knockout $\left(\mathrm{SERT}^{ \pm}\right)$dams that as pups have been exposed to early life stress (early life stress in dams, ELSD), resulting in depressive-like behavior (Houwing et al. 2019a). As reduced SERT expression has been associated with increased vulnerability to develop anxiety and depressive-like behavior after stressful life events (Caspi et al. 2003), but also with poorer response to FLX treatment in adults (Stevenson 2018), we expected offspring SERT genotype to interact with FLX and ELSD. Furthermore, as it has been shown that perinatal SSRI treatment can increase aggressive behavior in rodent offspring (Kiryanova et al. 2016; Kiryanova and Dyck 2014; Singh et al. 1998), we expected a similar increase in aggression toward an intruder after perinatal FLX treatment. In addition, we expected ELSD to reduce offspring aggressive behavior (Kinsley and Svare 1986; Kiryanova et al. 2016; Patin et al. 2005). When offspring is exposed to perinatal FLX and ELSD, we expect aggressive behavior in offspring to be normal (Kiryanova et al. 2016). Furthermore, since multiple studies show reduced sexual behavior after both SSRI exposure (Gouvêa et al. 2008; Maciag et al. 2006; Rayen et al. 2013) and prenatal stress (Hernández-Arteaga et al. 2016; Masterpasqua et al. 1976; Rhees and Fleming 1981), we similarly expected perinatal FLX treatment and ELSD, independently as well as combined, to reduce sexual behavior in the offspring.

\section{Materials and methods}

\section{Animals}

Wistar rats were maintained on a reversed 12-h light/dark cycle (lights off at 11:00 a.m.) with ad libitum access to food (RMH-B, AB Diets; Woerden, the Netherlands) and tap 
water. A wooden gnawing stick $(10 \times 2 \times 2 \mathrm{~cm})$ and nesting material (Enviro-dri $\left.{ }^{\circledR}\right)$ was provided for environmental enrichment. Animals were housed in Makrolon type 3 cages $(38.2 \times 22.0 \times 15.0 \mathrm{~cm})$ during individual housing or Makrolon type 4 cages $(55.6 \times 33.4 \times 19.5 \mathrm{~cm})$ during social housing. All breeding occurred in our own facility. All experimental procedures were approved by the Groningen University Committee of Animal experiments (DEC 6936A).

\section{Early life stress in dams}

Serotonin transporter knockout rats (Slc6a41Hubr) were bred crossing $\mathrm{SERT}^{+/}$females with $\mathrm{SERT}^{ \pm}$males, resulting in pups of three genotypes $\left(\mathrm{SERT}^{+/+}, \mathrm{SERT}^{ \pm}\right.$, and $\mathrm{SERT}^{-/-}$). These pups (future dams) were randomly assigned to either the control group (CTR) or the early life stress group (ELS). ELS consisted of maternally separating both male and female pups of all SERT genotypes as a whole litter for $6 \mathrm{~h}$ a day from postnatal day (PND)2-15. CTR pups were taken away during this period for 15 min and handled briefly. At PND21, pups were weaned and socially housed with same-treated, same sex pups from different litters. When adult, SERT ${ }^{ \pm}$females exposed to ELS show depressive-like behavior (Houwing et al. 2019a) and were therefore used as an animal model for maternal depression.

\section{Perinatal fluoxetine treatment}

In total, 85 (33 CTR and $52 \mathrm{ELS}$ ) female SERT ${ }^{ \pm}$and 47 male $\mathrm{SERT}^{+/+}$rats were used for breeding. Female estrus was determined by measuring vaginal wall impedance (model MK11, Muromachi, Tokyo, Japan) and followed by housing the receptive female together with a male for $24 \mathrm{~h}$ (gestational day 0, G0). CTR and ELS SERT ${ }^{ \pm}$females were randomly assigned to the vehicle-treated (VEH) or fluoxetine-treated (FLX) group. Using oral gavage, dams were treated daily with VEH (methylcellulose 1\%, Sigma-Aldrich Chemie BV, Zwijndrecht, the Netherlands) or FLX (10 mg/kg; Pharmachemie BV, the Netherlands) injected at a volume of $5 \mathrm{~mL} / \mathrm{kg}$ from G0 until weaning of the pups at PND21. For oral gavage, flexible PVC feeding tubes (Vygon, Valkenswaard, the Netherlands) were used without restraining the animals, thus minimizing stress. To determine the exact dosing volume, dams were weighed daily. Four groups of dams were used: (1) CTR dams + VEH treatment (CTRVEH) $(n=11)$, (2) CTR dams + FLX treatment (CTR-FLX) $(n=22)$, (3) ELS in dams + VEH treatment (ELSD-VEH) $(n=15)$, and (4) ELS in dams + FLX treatment (ELSDFLX) $(n=37)$. Dams were checked twice a day for pup delivery. Pregnancy outcomes of the dams and litter characteristics have been reported elsewhere (Houwing et al. 2019b). On PND21, pups were weaned, and ears were punched for individual recognition and genotyping (El Aidy et al. 2017).
SERT $^{+/+}$and SERT ${ }^{ \pm}$offspring were housed with 3-5 sametreated, same-sex animals from different litters while mixing genotypes. Due to unexpected high mortality rates in dams and offspring from FLX groups (Houwing et al. 2019b), offspring from three (aggressive behavior) or even four (sexual behavior) batches were needed to complete this study. In addition, because of the reduced survival of FLX pups, $4.7 \%$ of the litters (batch 1 to 3 ) up to five pups per litter were used. No litter effects were found.

\section{Behavioral testing}

Both SERT ${ }^{+/+}$and $\mathrm{SERT}^{ \pm}$male offspring were tested for aggressive and sexual behavior during adulthood. Different batches of males were used for aggressive and sexual behavior. Males used for aggressive behavior were previously tested for juvenile play (4-5 weeks of age) and social interaction (10-12 weeks of age) (Houwing et al. 2019b). Males used for sexual behavior were previously tested for affective behavior, including the open field test (16 weeks of age), the forced swim test (26 weeks of age), and the sucrose preference test (27-30 weeks of age) (Houwing et al., in preparation). Testing occurred between 12:00 p.m. and 5:00 p.m. during the active dark phase of the animals. Behavior was manually scored and analyzed by one observer blind to treatment using The Observer XT version 11.0 (Noldus Information Technology B.V., Wageningen, The Netherlands).

\section{Aggressive behavior}

Male (14-16 weeks old) offspring $\left(\right.$ SERT $^{+/+}$: CTR-VEH $n=$ $11, \mathrm{CTR}-\mathrm{FLX} n=11, \mathrm{ELSD}-\mathrm{VEH} n=11, \mathrm{ELSD}-\mathrm{FLX} n=10$. SERT $^{ \pm}$: CTR-VEH $n=11$, CTR-FLX $n=11$, ELSD-VEH $n=9$, ELSD-FLX $n=11$ ) were tested for aggressive behavior in the resident-intruder test (Koolhaas et al. 2013). Male residents were paired with an adult non-experimental SERT ${ }^{+/+}$ female and housed in a large cage $(80 \times 55 \times 50 \mathrm{~cm})$ for 1 week to habituate and establish territorial behavior. Females were oviduct ligated 2 weeks before the habituation period, to prevent pregnancy and the development of maternal aggression while keeping hormonal regulation intact. Pairs were housed with a polycarbonate tunnel and 2 wooden gnawing sticks $(10 \times 2 \times 2 \mathrm{~cm})$ for enrichment, while food (RMH-B, AB Diets; Woerden, the Netherlands), and tap water were present ad libitum. Cages were covered with a $2-\mathrm{cm}$ layer of Aspen wood chip bedding and were not cleaned during the habituation and testing period. Females and enrichment were removed at least $1 \mathrm{~h}$ before testing, and testing occurred under dim light conditions (10 lx). Residents received three training sessions followed by a test session on consecutive days. During training, an unfamiliar nonexperimental male $\mathrm{SERT}^{+/+}$rat (intruder) was introduced into the resident's cage, and the latency to attack the intruder by 
biting was observed. After the first biting attack, or when no attack occurred within $10 \mathrm{~min}$, the intruder was removed. Residents and intruders were weighed prior to habituation to ensure that intruders were lighter than residents. Intruders were only used once a day, and each resident received an unfamiliar intruder for each training and test session. During the test session, aggressive behavior of the resident was recorded on video for $10 \mathrm{~min}$ after the first attack. If residents did not attack the intruder, the first $10 \mathrm{~min}$ of the video were scored. The frequency and duration of the following behaviors of the resident were manually scored from the video recordings: (1) offensive behavior (lateral threat, upright posture, clinch attack, keep down, and chasing), (2) social behavior (moving toward/following, social sniffing, social grooming), (3) defensive behavior (keep off and submissive behavior), and (4) non-social behavior (non-social exploration, rearing, inactivity, and self-grooming). Furthermore, the latency to attack the intruder was scored. In addition, we calculated the proportion of animals attacking the intruder during the test session. Behavioral measures were converted into percentage of total time spent on the behavior.

\section{Sexual behavior}

Male (32 weeks old) offspring (SERT ${ }^{+/+}:$CTR-VEH $n=10$, CTR-FLX $n=10$, ELSD-VEH $n=11$, ELSD-FLX $n=10$, SERT $^{ \pm}$: CTR-VEH $n=10$, CTR-FLX $n=10$, ELSD-VEH $n=11$, ELSD-FLX $n=11$ ) were assessed for sexual behavior in $45 \times 30 \times 50 \mathrm{~cm}$ Phenotyper cages (Noldus Information Technology B.V., Wageningen, The Netherlands) under red light conditions. Cages were covered with a 2-cm layer of Aspen wood chip bedding and were not cleaned during the testing period. Adult female $\mathrm{SERT}^{+/+}$rats were used as stimulus animals, and estrous was induced with a single s.c. injection of $50 \mu \mathrm{g}$ estradiol benzoate (dissolved in sesame oil saturated with lecithin) 36-42 $\mathrm{h}$ prior to testing (Olivier et al. 2011). First, males were allowed to habituate to the test cage for $10 \mathrm{~min}$. Subsequently, a receptive female was introduced, and sexual behavior was recorded on video for $30 \mathrm{~min}$. To ensure the male was able to interact with a receptive and willing female, female behavior was observed within the first $5 \mathrm{~min}$ of the test. If she repeatedly rejected the male (e.g., kicking, turning around to prevent mounting), she was replaced. Training of sexual behavior occurred once a week for 6 consecutive weeks. Sexual behavior was recorded on video tape in week 7, when the animals were sexually experienced. The frequency and latency of the following male copulatory behaviors were manually scored from video: mounts, intromissions, and ejaculations. Mounting and intromission frequency of the first ejaculatory series (frequency up until the first ejaculation) were used. Copulatory efficiency was calculated by determining the intromission ratio: number of intromissions/(number of intromissions + number of mounts)*100.

\section{Statistical analysis}

Data was analyzed using the Statistical Package for the Social Sciences (SPSS) software version 22 (SPPS Inc., IBM SPSS Statistics, Chicago). Upon non-parametric distribution, data was transformed to approach parametric distribution for use in statistical analysis. For behavior in the resident-intruder test, offensive and defensive behaviors were log transformed. Regarding sexual behavior, a square root transformation was performed for mounting, intromission, and ejaculation frequency. In addition, latency of copulatory behaviors was log transformed. All other behavioral parameters approached parametric distribution.For both aggressive and sexual behavior, a three-way ANOVA was performed to determine main and/or interaction effects of FLX, ELSD, and genotype. There were no significant 3-way interactions found for any of the behavioral parameters analyzed $(p>.05)$. When found, twoway interactions and main effects are described in the results. For the proportion of animals attacking, a $\chi^{2}$ analysis was performed. Upon significant main and/or interaction effects, post hoc testing was performed using Fisher's LSD to correct for multiple comparisons. All statistics were two-tailed with values of $p \leq .05$ being considered significant. When adding litter as a factor in our ANOVA, no significant interactions between litter and other factors were found. All data and all figures are presented as mean \pm standard error of the mean (SEM). Outliers were not removed, and individual data points are shown in each figure.

\section{Results}

\section{Aggressive behavior}

Effects of perinatal FLX treatment and ELSD on aggressive behavior in $\mathrm{SERT}^{+/+}$and $\mathrm{SERT}^{ \pm}$male offspring were assessed using the resident intruder test. Perinatal FLX treatment significantly reduced offensive behavior in the offspring, regardless of ELSD and genotype $\left(\mathrm{F}_{(1,77)}=17.686, p<.001\right.$, Fig. 1a). Post hoc testing revealed that SERT ${ }^{+/+}$CTR-FLX offspring showed less offensive behavior compared with SERT $^{+/+}$CTR-VEH offspring $(p<.01$, Fig. 1a). Similarly, SERT $^{+/+}$ELSD-FLX offspring displayed less offensive behavior compared with SERT $^{+/+}$ELSD-VEH offspring $(p<.01$, Fig. 1a). Furthermore, ELSD significantly increased offensive behavior in the offspring, regardless of perinatal FLX treatment and genotype $\left(\mathrm{F}_{(1,77)}=7.389, p<.01\right.$, Fig. 1a). In addition, an interaction between ELSD and genotype was found $\left(\mathrm{F}_{(1,77)}=4.786, p<.05\right)$, with both $\mathrm{SERT}^{ \pm}$ ELSD-VEH and SERT ${ }^{ \pm}$ELSD-FLX offspring showing 
Fig. 1 Effects of perinatal FLX treatment and ELSD on various behaviors of adult male offspring in the resident intruder test. Shown are offensive behavior (a), defensive behavior (b), social behavior (c), non-social behavior (d), attack latency (e), and proportion of animals attacking (f) during the test session.

Figures show mean \pm SEM. ${ }^{*} p \leq .05, * * p<.01, \$ \$ p<.01$ (vs SERT $^{+/+}$CTR-VEH). $n=9-12$ per group

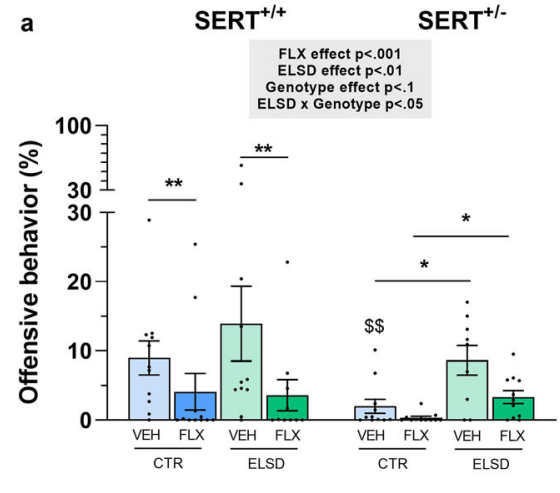

c

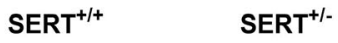

Genotype effect $p<.05$

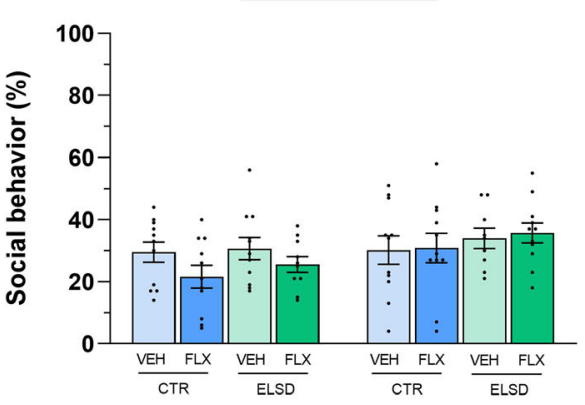

e

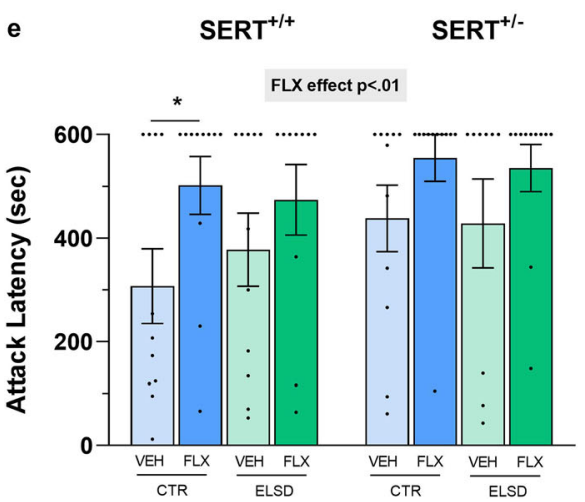

b

SERT $^{+/+}$

SERT $^{+/-}$

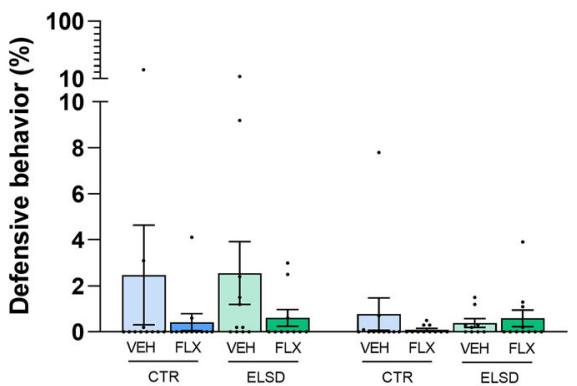

d $\quad \mathrm{SERT}^{+/+} \quad \mathrm{SERT}^{+/-}$
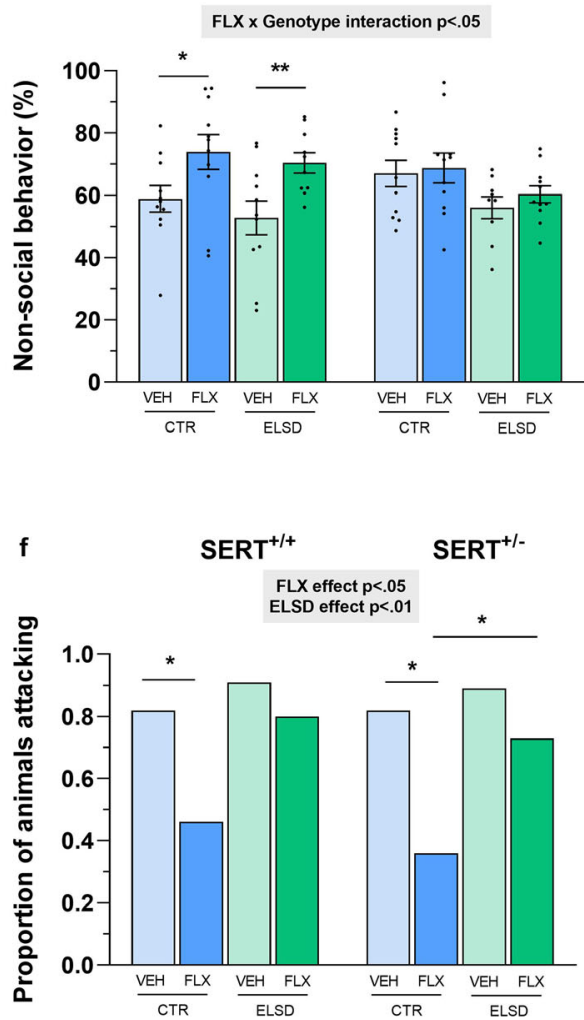

significantly increased offensive behavior compared with SERT $^{ \pm}$offspring from same-treated CTR dams (CTR-VEH vs. ELSD-VEH: $p<.05$, CTR-FLX vs. ELSD-FLX: $p<.05)$, while SERT $^{+/+}$offspring were not affected (Fig. 1a). Also, a tendency toward a main effect of genotype was found with $\mathrm{SERT}^{ \pm}$offspring showing less offensive behavior compared with SERT ${ }^{+/+}$offspring $\left(\mathrm{F}_{(1,77)}=3.264\right.$, $p=.08$, Fig. 1a). More specifically, post hoc testing revealed that SERT $^{ \pm}$CTR-VEH offspring showed less offensive behavior compared with $\mathrm{SERT}^{+/+} \mathrm{CTR}-\mathrm{VEH}$ offspring $(p<.01$, Fig. 1a). Defensive behavior was not affected by ELSD, perinatal FLX treatment, or offspring genotype $(p>.05$, Fig. 1b). When looking into non-aggressive social behavior, a significant main effect of genotype was found with SERT $^{ \pm}$offspring showing increased social behavior compared with SERT $^{+/+}$offspring, regardless of perinatal FLX treatment and $\operatorname{ELSD}\left(\mathrm{F}_{(1,77)}=4.901, p<.05\right.$, Fig. 1c $)$. Regarding non-social behavior, a significant interaction between perinatal FLX treatment and genotype was found $\left(\mathrm{F}_{(1,77)}=4.578\right.$, $p<.05$, Fig. 1d). Both SERT ${ }^{+/+}$CTR-FLX and SERT ${ }^{+/+}$ELSDFLX offspring showed increased non-social behavior compared with VEH-treated counterparts (CTR-FLX vs. CTR-VEH: $p<.05$, ELSD-FLX vs. ELSD-VEH: $p<.01$ ), while perinatal FLX treatment did not affect non-social behavior in $\mathrm{SERT}^{ \pm}$offspring (Fig. 1d). For the attack latency, there was a significant main effect found of perinatal FLX treatment, with perinatal FLX 
treatment increasing the latency to attack the intruder, regardless of ELSD and genotype $\left(\mathrm{F}_{(1,77)}=8.101, p<.01\right.$, Fig. 1e). Post hoc analysis showed that SERT ${ }^{+/+}$CTR-FLX offspring had a higher latency to attack the intruder than SERT ${ }^{+/+}$CTR-VEH offspring $(p<.05$, Fig. 1e). Regarding the proportion of animals attacking the intruder, a significant main effect of perinatal FLX treatment was found, with a smaller proportion of animals attacking, regardless of ELSD and genotype $\left(\chi_{(1,85)}^{2}=7.973, p<.01\right.$, Fig. 1f). Also, a significant main effect of ELSD was found, with a higher proportion of animals attacking the intruder, regardless of perinatal FLX treatment and genotype $\left(\chi_{(1,85)}^{2}=4.870\right.$, $p<.05$, Fig. 1f). Post hoc testing revealed that a smaller proportion of SERT ${ }^{+/+}$CTR-FLX offspring attacked the intruder than SERT $^{+/+}$CTR-VEH offspring ( $p=.05$, Fig. 1f). Also, a significant smaller proportion of SERT ${ }^{ \pm}$CTR-FLX offspring attacked the intruder compared with both SERT ${ }^{ \pm}$CTR-VEH $(p<.05)$ and SERT $^{ \pm}$ELSD-FLX offspring ( $p=.05$ ).

\section{Sexual behavior}

Effects of perinatal FLX treatment and ELSD on male copulatory behaviors in $\mathrm{SERT}^{+/+}$and $\mathrm{SERT}^{ \pm}$offspring were assessed after 6 weeks of training, when animals were sexually experienced. A tendency toward an interaction between perinatal FLX treatment and genotype was found for mounting frequency $\left(\mathrm{F}_{(1,75)}=3.671, p=.06\right)$, with post hoc testing revealing that SERT $^{+/+}$CTR-FLX offspring showed less mounting behavior compared with SERT $^{+/+}$CTR-VEH offspring $(p<.05$, Fig. 2a). When looking at the latency until the first mount, a significant main effect of genotype was found, with SERT ${ }^{ \pm}$offspring showing a reduced mounting latency compared with $\mathrm{SERT}^{+/+}$offspring, regardless of perinatal FLX treatment and ELSD $\left(\mathrm{F}_{(1,75)}=3.923, p=.05\right.$, Fig. $\left.2 b\right)$. However, post hoc comparisons show no genotype differences between offspring groups. For the number of intromissions, again a significant main effect of genotype was found, with SERT $^{ \pm}$offspring displaying an increased number of intromissions compared with $\mathrm{SERT}^{+/+}$offspring, regardless of perinatal FLX treatment and ELSD $\left(\mathrm{F}_{(1,75)}=5.702, p<.05\right.$, Fig. 2c). More specifically, post hoc analysis showed that SERT $^{ \pm}$CTR-FLX offspring performed more intromissions than SERT ${ }^{+/}$CTR-FLX offspring $(p<.05$, Fig. 2c). Also, for the intromission ratio, a significant main effect of genotype was found, with SERT $^{ \pm}$offspring showing a higher intromission ratio than $\mathrm{SERT}^{+/+}$offspring, regardless of perinatal FLX treatment and ELSD $\left(\mathrm{F}_{(1,75)}=9.924\right.$, $p<.01$, Fig. 2d). Post hoc analysis revealed that SERT ${ }^{ \pm}$ ELSD-VEH offspring had a higher intromission ratio than $\mathrm{SERT}^{+/+}$ELSD-VEH offspring, suggesting higher copulatory efficiency (Fig. 2d). No other significant main and/or interaction effects were found for the ejaculation frequency (Fig. 2e) and other behavioral parameters for copulatory behavior. Between batches, animals significantly differed in their sexual performance (ejaculation frequency: $\left.\mathrm{F}_{(3,82)}=14.927, p<.001\right)$. With an average ejaculation frequency below 1 , animals in the second $(0.53 \pm 0.48)$, third $(0.90 \pm 0.21)$, and fourth $(0.64 \pm 0.29)$ batch were poor sexual performers and differed significantly from the first batch $(2.48 \pm 0.20, p<.001)$, where animals performed normally.

\section{Discussion}

In this study, we sought to explore the effects of perinatal FLX treatment and dams' early life stress history, both separately and combined, on aggressive and sexual behavior in adult $\mathrm{SERT}^{+/+}$and $\mathrm{SERT}^{ \pm}$male offspring. Our main findings demonstrate that perinatal FLX treatment lowered aggressive behavior, especially in $\mathrm{SERT}^{+/+}$offspring, while early life stress in dams (ELSD) heightened aggressive behavior in SERT ${ }^{ \pm}$ offspring. Perinatal FLX treatment caused mild effects on sexual behavior of SERT ${ }^{+/+}$offspring, while ELSD did not affect sexual behavior. Finally, we found genotype-specific effects. SERT $^{ \pm}$offspring showed less offensive but more sexual activity compared with $\mathrm{SERT}^{+/+}$rats. Altogether, these data show that both perinatal FLX treatment and ELSD genotype dependently affect social behavior in the offspring.

\section{Effects of perinatal FLX treatment on offspring aggressive behavior}

Against our expectations, FLX treatment in healthy dams reduced aggressive behavior in $\mathrm{SERT}^{+/+}$offspring. This was reflected in less time spent in offensive behavior, an increased attack latency, and a smaller proportion of animals attacking the intruder. Our findings contradict both clinical and preclinical data, where perinatal SSRI treatment has been associated with an increase in aggressive behavior. In humans, SSRI treatment during pregnancy has been associated with increased externalizing behaviors such as aggression or defiant behavior in 4-year-olds (Oberlander et al. 2007). Similarly, preclinical studies show that perinatal FLX treatment has been associated with an increase in male aggressive behavior during adulthood. FLX treatment during the last week of pregnancy increased the number of foot shock-induced aggressive fighting bouts in adolescent rat offspring (Singh et al. 1998). FLX treatment during the entire pregnancy increased the number and duration of aggressive attacks in adult male mice (Svirsky et al. 2016). In addition, Kiryanova and Dyck (2014) found that perinatal FLX treatment from G15PND21 increased the proportion of mice attacking an intruder, while other parameters of aggressive behavior were unaffected. Related to the reduced aggression levels we found in the present study, Yu et al. (2014) showed that male mice exposed 
Fig. 2 Effects of perinatal FLX treatment and ELSD on male offspring copulatory behaviors. Shown are the mounting frequency (a), mounting latency (b), intromission frequency (c), intromission ratio (d), and the ejaculation frequency (e).

Figures show mean \pm SEM. $* p \leq$ $0.05, \$ p \leq 0.05$ (vs. $\mathrm{SERT}^{+/+}$), \$\$ $p<0.01$ (vs. SERT ${ }^{+/+}$). $n=10-11$ per group a

SERT $^{+/+}$

SERT $^{+/-}$

FLX $\times$ Genotype effect $p<.1$

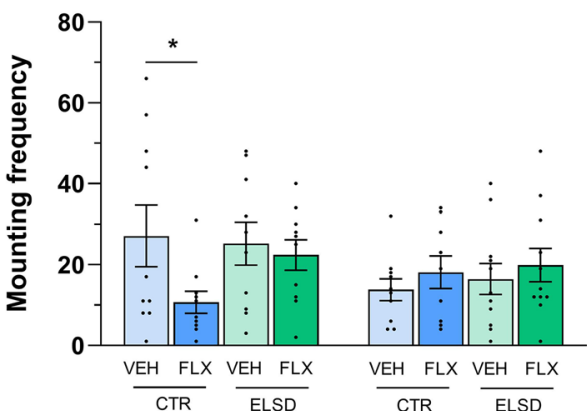

C

SERT $^{+/+}$

SERT $^{+/-}$

Genotype effect $p<.05$

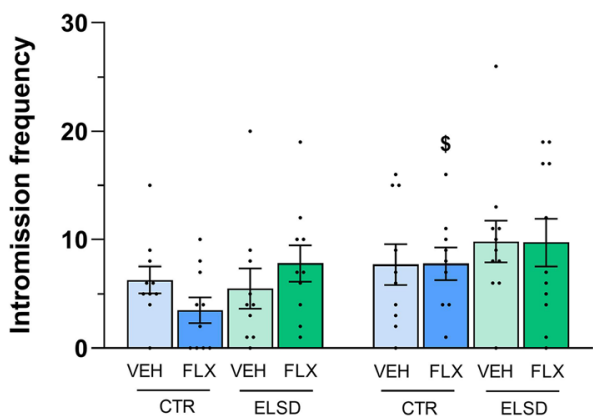

e $\quad \mathrm{SERT}^{+/+}$

SERT $^{+/-}$

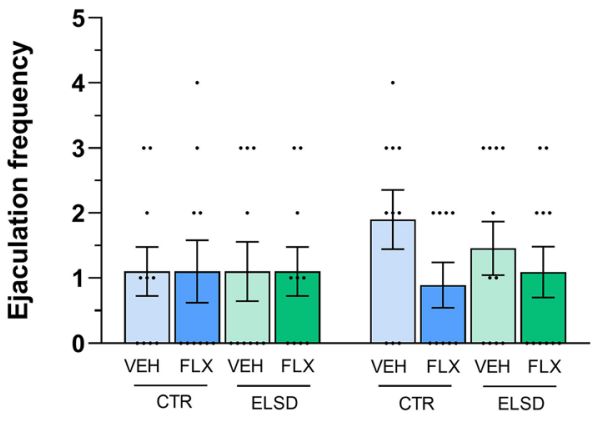

b

SERT $^{+/+}$

SERT $^{+/-}$

Genotype effect $p=.05$

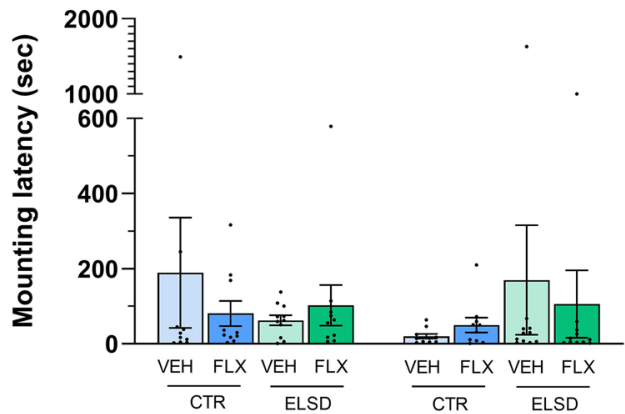

d

SERT $^{+/+}$

SERT $^{+/-}$

Genotype effect $p<.01$

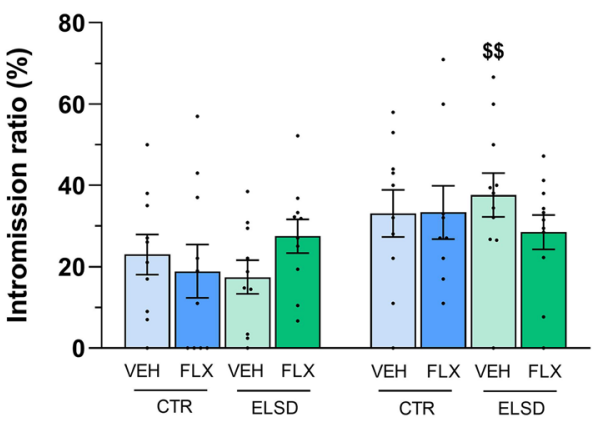

to FLX during PND2-PND21 displayed reduced aggressive behavior toward an intruder, even failing to display some characteristic aggressive behaviors such as biting and tail rattling. Furthermore, Lisboa et al. (2007) exposed male mice offspring to FLX throughout pregnancy and lactation (G0PND21), similar to our study, and found a trend toward an increased attack latency but no effects on number of attacks or attack duration in adult male offspring. All taken together, it seems that prenatal FLX treatment increases offspring aggressive behavior, while postnatal FLX exposure reduces aggressive behavior. However, when FLX is given during both the pre- and postnatal period, results on aggressive behavior in the offspring are inconsistent. One possible explanation for the found reduction in offspring aggressive behavior is that our animals are less motivated to participate in social interactions. We previously found that the animals used in this study engage less in juvenile social play behavior and adult social interactions (Houwing et al. 2019b). Indeed, in the present study, we found that FLX-exposed offspring displayed more non-social behaviors, such as non-social exploration. At the same time, FLX-exposed offspring spent a similar amount of time resting, indicating that FLX-exposed offspring were not simply less active than VEH-exposed offspring (data not shown). 
Interestingly, reduced aggressive behavior after developmental FLX exposure corresponds with findings in SERT ${ }^{-/}$ - rodents, who similarly display reduced levels of aggression. Both SSRI exposure and diminished SERT expression result in elevated extracellular serotonin levels. Whereas $\mathrm{SERT}^{-1-}$ rodents have chronically elevated 5-HT levels and display reduced aggressive behavior compared with SERT $^{+/+}$rodents, SERT $^{ \pm}$rodents have similar extracellular 5-HT levels to SERT $^{+/+}$(reviewed by Houwing et al. 2017). Interestingly, we observed reduced offensive behavior in SERT $^{ \pm}$CTR-VEH offspring when compared with $\mathrm{SERT}^{+/+}$offspring, while it is often reported that $\mathrm{SERT}^{+/+}$ and $\mathrm{SERT}^{ \pm}$rodents show no differences in behavior. Perhaps these rats function similar under normal conditions but may respond differently under stressful situations. Overall, we found reduced aggressive behavior when animals are exposed to FLX. Discrepancies between studies in aggressive behavior could be attributed to several factors, including the variation in behavioral tests to measure aggressive behavior, species used, FLX dose, and treatment period (pre- versus postnatal) and treatment duration (days versus weeks).

\section{Effects of perinatal FLX treatment on offspring sexual behavior}

Regarding sexual behavior, our findings demonstrated that male CTR offspring, but not ELSD offspring, exposed to perinatal FLX show reduced mounting behavior, specifically in $\mathrm{SERT}^{+/+}$offspring. However, other copulatory behaviors such as intromissions, ejaculations, and the intromission ratio were not affected, suggesting that sexual motivation is only slightly reduced, while leaving sexual performance intact. In humans, not much is known about SSRI treatment during pregnancy and lactation and the effects it can have on offspring sexual behavior later in life, but preclinical studies found that early SSRI exposure can lead to reduced copulatory behaviors in rodents. For example, when rat pups are treated with the SSRI citalopram from PND8-PND21, the number of mounts, intromissions, and ejaculations are reduced in both naïve and sexually experienced male rats (Harris et al. 2012; Maciag et al. 2006; Rodriguez-Porcel et al. 2011). Similarly, FLX exposure during the postnatal period reduced sexual motivation and performance in adult male rats (Rayen et al. 2013; Rodriguez-Porcel et al. 2011). However, when animals were exposed to FLX during the prenatal period, or throughout the pre- and postnatal period, male copulatory behavior was unaffected in mice (Gouvêa et al. 2008) and rats (Cagiano et al. 2008; Olivier et al. 2011; Vieira et al. 2013). All in all, the present study shows that perinatal FLX exposure did not affect overall sexual performance, although a small decrease was found in sexual motivation.

\section{Effects of ELSD on offspring aggressive and sexual behavior}

Against our expectations, ELSD increased aggressive behavior in male $\mathrm{SERT}^{ \pm}$offspring, which was reflected in increased offensive behavior toward an intruder. However, when these pups were also exposed to FLX, aggression levels were comparable with the control animals. Our findings contradict previous literature where prenatal stress reduced aggressive behavior in male offspring (Kinsley and Svare 1986; Kiryanova et al. 2016; Patin et al. 2005). While prenatal stress reduces aggressive behavior in offspring, stressors during the early postnatal period, such as maternal separation, have the potential to increase inter-male aggression in rats (Veenema et al. 2006) and maternal aggression in mice (Veenema et al. 2007). Although the offspring in the present study were not exposed to maternal separation, their mothers were exposed to maternal separation. Because transgenerational effects of early life stress have been reported in both humans (Esteves et al. 2019) and rodents (Weiss et al. 2011), our results in the offspring may be the result of transgenerational effects of early life stress in our dams. One of the mechanisms responsible for the potential transgenerational effects on aggressive behavior is through physiological changes in the dam. Both prenatal stress (Rakers et al. 2017) and maternal separation can induce maternal cortisol (or corticosterone (CORT) in rodents) release which can be transferred to the fetus via the placenta to induce changes in the fetal hypothalamic-pituitary-adrenal axis and subsequent behavioral alterations later in life. In fact, maternal separation for $3 \mathrm{~h}$ a day on PND1-14 can increase basal CORT levels and other regulators of the HPA-axis such as hypothalamic CRF mRNA levels in juvenile (Veenema and Neumann 2009) and adult (Plotsky and Meaney 1993) male rats. However, $6 \mathrm{~h}$ of maternal separation from PND2-15 in our dams did not affect basal CORT levels prior to breeding (Houwing et al. 2019a) and was not measured during pregnancy or in the offspring. Even so, a depressive-like phenotype during pregnancy is likely to induce elevated stress levels in the dams, and maternal-fetal stress transfer is likely to occur (Brummelte and Galea 2010), but we do not know whether maternal or fetal CORT levels are correlated with aggression levels in the offspring. Whether the dams exposed to early life stress indeed show more maternal aggression and if these effects are transferred to the offspring remain to be further investigated. Another possibility for the increased aggression found in our offspring is due to altered maternal care of the dam. Prenatal stress can either increase (Rayen et al. 2011), reduce (Smith et al. 2004), or have no effect on maternal care of the dam (Gemmel et al. 2018b; Kiryanova et al. 2016; Pawluski et al. 2012a, b). Predictive maternal separation can increase maternal care of the dam (Franklin et al. 2010), and there is evidence for the behavioral transmission of maternal care behavior from dams to female offspring (reviewed by 
Champagne 2008). Whether the observed increase in aggressive behavior in offspring of mothers exposed to early life stress are associated with altered maternal care levels remains to be investigated. Regarding sexual behavior, no effects of ELSD was found on copulatory behaviors of male offspring. These findings are opposed to previous studies that have repeatedly found prenatal stress to impair male sexual behavior (Gerardin et al. 2005; Hernández-Arteaga et al. 2016; Masterpasqua et al. 1976; Wang et al. 2006) but are similar to Rayen and colleagues who found no effects of prenatal stress on male (Rayen et al. 2013) and female (Rayen et al. 2014) sexual performance. To our knowledge, the effects of ELSD on male sexual behavior in the next generation have not been documented. Clearly, more research is needed on the transgenerational effects of ELSD on sexual behavior performance of male offspring.

\section{Effects of offspring SERT genotype and its interaction with perinatal FLX treatment and ELSD}

Our results demonstrated that $\mathrm{SERT}^{ \pm}$males showed reduced offensive behavior and increased non-aggressive social behavior compared with $\mathrm{SERT}^{+/+}$offspring. Furthermore, $\mathrm{SERT}^{ \pm}$males showed a decreased mounting latency and increased intromission frequency and intromission ratio compared with $\mathrm{SERT}^{+/+}$males, suggesting increased sexual motivation and copulatory efficiency, without affecting sexual performance. This is surprising, as differences in aggressive and sexual behavior between $\mathrm{SERT}^{+/+}$and $\mathrm{SERT}^{ \pm}$rodents have not been found before (Chan et al. 2011; Esquivel-Franco et al. 2018; Homberg et al. 2007), and SERT ${ }^{-/-}$rodents usually show impaired sexual behavior (Chan et al. 2011). When SERT $^{ \pm}$offspring were split for treatment groups, not many differences were found. In fact, when just looking at SERT ${ }^{ \pm}$ CTR-VEH offspring, which is the most comparable group to previous literature, we only find offensive behavior to be reduced while sexual behavior is unaltered.

Next, we were interested in a possible interaction between offspring SERT genotype and perinatal FLX treatment. In primates, having the short (S) allele for a polymorphism in the promoter region of the SERT gene results in lower SERT expression. Lower SERT expression has been associated with a poorer response to FLX treatment (Stevenson 2018). Keeping this in mind, the reduced SERT expression in our SERT ${ }^{ \pm}$rats might play a role in the response to perinatal SSRI exposure as well. Interestingly, effects of perinatal FLX treatment on aggressive behavior were primarily observed in $\mathrm{SERT}^{+/+}$offspring and almost completely absent in $\mathrm{SERT}^{ \pm}$ offspring. Our study even shows that levels of offensive behavior in $\mathrm{SERT}^{ \pm}$offspring were quite low to begin with, which may have concealed effects of perinatal FLX treatment. When it comes to sexual behavior, perinatal FLX treatment and SERT genotype interacted to decrease mounting in
$\mathrm{SERT}^{+/+}$offspring only. Consequently, our findings suggest that $\mathrm{SERT}^{ \pm}$offspring are less sensitive to developmental FLX exposure than $\mathrm{SERT}^{+/+}$offspring. Furthermore, we found an interaction between SERT genotype and ELSD on offspring offensive behavior. In fact, ELSD increased offensive behavior in $\mathrm{SERT}^{ \pm}$offspring only, suggesting that $\mathrm{SERT}^{ \pm}$male offspring are more sensitive to ELSD than SERT ${ }^{+/+}$offspring. Our results seem in line with the finding that human S-allele carriers that are exposed to stressful life events are more susceptible to stress and have an increased risk to develop mental disorders like major depression. All in all, our results indicate that $\mathrm{SERT}^{ \pm}$offspring are less sensitive to FLX-induced effects on sexual behavior while being more sensitive to ELSDinduced effects on aggressive behavior.

\section{Limitations and future perspectives}

After analyzing our data, we discovered that a large part of our males used for sexual behavior were poor sexual performers. In fact, $46 \%$ of our male offspring did not ejaculate at all during the 30-min test session, while sexually experienced $\mathrm{SERT}^{+/+}$and $\mathrm{SERT}^{ \pm}$Wistar rats usually ejaculate on average 2.5 times (Chan et al. 2011). Poor sexual performers were present in all treatment groups. However, frequencies of other copulatory behaviors were within normal range. We also discovered that there were differences in the 4 batches tested. In fact, the $2 \mathrm{nd}$, 3rd, and 4th batches were poor sexual performers, while the 1st batch performed normally. Further analysis did not show an interaction between batch and perinatal FLX treatment, ELSD, or genotype on any of the behavioral parameters tested and indicated a main effect of batch only. Even so, this difference in sexual performance between batches could have concealed effects of FLX, ELSD, or genotype on sexual behavior, which could be present when sexual performance is normal across all batches. Factors responsible for poor sexual performance in these batches might be related to subtle changes during the experiments such as a different season or having a different experimenter that performed testing with each batch (although all data were scored by the same experimenter), but the exact factor remains unknown. Future studies should preferably use one batch of animals, where at least CTR animals should on average display normal sexual performance. Another limitation of the study is that all animals underwent other behavioral tests prior to testing for aggressive and sexual behavior. Being exposed to a behavioral test battery is known to potentially change behavioral outcomes compared with animals naïve for testing experience (McIlwain et al. 2001). The animals used for aggressive behavior were previously exposed to (playful) friendly social interactions at juvenile and adult age, which we believe will have minimal effects on aggressive behavior. Animals used for sexual behavior previously underwent testing for anxiety and depressive-like behavior, which could 
potentially affect behavioral outcomes. Furthermore, sexual behavior was trained once a week for 7 consecutive weeks. Thus, the behavior reported here is of experienced males, which is comparable with previous studies testing males after several sexual training sessions (Olivier et al. 2011; Rodriguez-Porcel et al. 2011).

Furthermore, the present study investigated male aggressive and sexual behavior only. Previously, it has been shown that effects of developmental FLX exposure can have opposite effects on male and female sexual behavior (Rayen et al. 2013, 2014). Rayen et al. (2013) showed that early postnatal FLX exposure reduces copulatory behaviors in males, while female proceptive and receptive behaviors were facilitated (Rayen et al. 2014). In addition, potential alterations in female aggression, for example, maternal aggression, after perinatal FLX exposure and maternal stress have not been investigated before. Therefore, future research should include both sexes when looking at the effects of perinatal FLX treatment and maternal stress on offspring aggressive and sexual behavior.

\section{Conclusions}

In summary, our research showed that perinatal FLX treatment has profound long-term effects on male aggressive behavior, while having little impact on male sexual behavior. Our results add to the increasing amount of clinical and preclinical literature linking perinatal SSRI treatment to alter neurobehavioral development in the offspring and suggest a role for offspring SERT genotype. However, the underlying maternal depression might also contribute to long-term neurobehavioral alterations in the offspring. The risks and benefits of SSRI use during pregnancy therefore need further investigation, and more studies should include a translational animal model of maternal depression, to provide better insights into the effects in the offspring. Furthermore, future studies should include both male and female offspring to determine implications for behavioral development later in life. Altogether, understanding the effects of SSRI use during pregnancy and the postnatal period can help depressed women to make more informed decisions about the initiation or continuation of antidepressant treatment during pregnancy.

Acknowledgments We thank Christa Reitzema-Klein and Linda PalsRobben for their assistance with female oviduct ligations, Christa Reitzema-Klein for assistance with the resident-intruder test, and Judith Swart and Jolien de Weerd for their assistance with sexual behavior experiments.

Author contributions Study design, J.O., D.H., and S. de B.; Dam treatment and animal caretaking, D.H., J.O., and A.R.; behavioral experiments, D.H., K.S., E.S., and C.A.; genotyping, D.H. and A.R.; manual scoring of observational data, D.H. and D. E-F.; data analysis, D.H.; writing, original draft preparation, D.H.; writing, review, and editing,
J.O., S. de B., A.R., and D.H.; supervision, J.O. All authors have finalized and approved the content.

Funding information This work was supported by the NARSAD young investigator grant from the Brain and Behavior Research Foundation (Grant No. 25206) and by the European Union's Horizon 2020 Research and Innovation Programme under the Marie SkłodowskaCurie (Grant Agreement No. 660152).

\section{Compliance with ethical standards}

Conflict of interest The authors declare that they have no conflict of interest.

Abbreviations CORT, Corticosterone; CTR, Control; ELS, Early life stress; ELSD, Early life stress in dams; FLX, Fluoxetine; G, Gestational day; MS, Maternal separation; PND, Postnatal day; SERT, Serotonin transporter; SSRI, Selective serotonin reuptake inhibitor; VEH, Vehicle

Open Access This article is licensed under a Creative Commons Attribution 4.0 International License, which permits use, sharing, adaptation, distribution and reproduction in any medium or format, as long as you give appropriate credit to the original author(s) and the source, provide a link to the Creative Commons licence, and indicate if changes were made. The images or other third party material in this article are included in the article's Creative Commons licence, unless indicated otherwise in a credit line to the material. If material is not included in the article's Creative Commons licence and your intended use is not permitted by statutory regulation or exceeds the permitted use, you will need to obtain permission directly from the copyright holder. To view a copy of this licence, visit http://creativecommons.org/licenses/by/4.0/.

\section{References}

Anderson DJ (2012) Optogenetics, sex, and violence in the brain: implications for psychiatry. BPS 71:1081-1089. https://doi.org/10.1016/ j.biopsych.2011.11.012

Azmitia EC (2001) Modern views on an ancient chemical: serotonin effects on cell proliferation, maturation, and apoptosis. Brain Res Bull 56:413-424. https://doi.org/10.1016/S0361-9230(01)00614-1

Brown HK, Hussain-Shamsy N, Lunsky Y, Dennis CLE, Vigod SN (2017) The association between antenatal exposure to selective serotonin reuptake inhibitors and autism: a systematic review and meta-analysis. J Clin Psychiatry 78:e48-e58. https://doi.org/10. 4088/JCP.15r10194

Brummelte S, Galea LAM (2010) Depression during pregnancy and postpartum: contribution of stress and ovarian hormones. Prog Neuropsychopharmacol Biol Psychiatry 34:766-776. https://doi. org/10.1016/j.pnpbp.2009.09.006

Cagiano R, Flace P, Bera I, Maries L, Cioca G, Sabatini R, Benagiano V, Auteri P, Marzullo A, Vermesan D, Stefanelli R, Ambrosi G (2008) Neurofunctional effects in rats prenatally exposed to fluoxetine. Eur Rev Med Pharmacol Sci 12:137-148

Caspi A, Sugden K, Moffitt TE, Taylor A, Craig IW, Harrington H, McClay J, Mill J, Martin J, Braithwaite A, Poulton R (2003) Influence of life stress on depression: moderation by a polymorphism in the 5-HTT gene. Science 301:386-389. https://doi.org/ 10.1126/science. 1083968 
Champagne FA (2008) Epigenetic mechanisms and the transgenerational effects of maternal care. Front Neuroendocrinol 29:386-397. https:// doi.org/10.1016/j.yfrne.2008.03.003

Chan JSW, Snoeren EMS, Cuppen E, Waldinger MD, Olivier B, Oosting RS (2011) The serotonin transporter plays an important role in male sexual behavior: a study in serotonin transporter knockout rats. J Sex Med 8:97-108. https://doi.org/10.1111/j.1743-6109.2010.01961.x

El Aidy S, Ramsteijn AS, Dini-Andreote F, van Eijk R, Houwing DJ, Salles JF et al (2017) Serotonin transporter genotype modulates the gut microbiota composition in young rats, an effect augmented by early life stress. Front Cell Neurosci 11:1-12. https://doi.org/10. 3389/fncel.2017.00222

Esquivel-Franco DC, Olivier B, Waldinger MD, Gutiérrez-Ospina G, Olivier JDA (2018) Tramadol's inhibitory effects on sexual behavior: pharmacological studies in serotonin transporter knockout rats. Front Pharmacol 9:1-11. https://doi.org/10.3389/fphar.2018.00676

Esteves KC, Jones CW, Ph D, Wade M, Ph D, Callerame K et al (2019) Adverse childhood experiences: implications for offspring telomere length and psychopathology. Am J Psychiatry 177:1-11. https://doi. org/10.1176/appi.ajp.2019.18030335

Franklin TB, Russig H, Weiss IC, Grff J, Linder N, Michalon A et al (2010) Epigenetic transmission of the impact of early stress across generations. Biol Psychiatry 68:408-415. https://doi.org/10.1016/j. biopsych.2010.05.036

Gaspar P, Cases O, Maroteaux L (2003) The developmental role of serotonin: news from mouse molecular genetics. Nat Rev Neurosci 4: 1002-1012. https://doi.org/10.1038/nrn1256

Gemmel M, Bögi E, Ragan C, Hazlett M, Dubovicky M, van den Hove DL, Oberlander TF, Charlier TD, Pawluski JL (2018a) Perinatal selective serotonin reuptake inhibitor medication (SSRI) effects on social behaviors, neurodevelopment and the epigenome. Neurosci Biobehav Rev 85:102-116. https://doi.org/10.1016/j.neubiorev. 2017.04.023

Gemmel M, Kokras N, Dalla C, Pawluski JL (2018b) Perinatal fluoxetine prevents the effect of pre-gestational maternal stress on 5-HT in the PFC, but maternal stress has enduring effects on mPFC synaptic structure in offspring. Neuropharmacology 128:168-180. https:// doi.org/10.1016/j.neuropharm.2017.10.009

Gentile S (2005) SSRIs in pregnancy and lactation. CNS Drugs 19:623633. https://doi.org/10.2165/00023210-200519070-00004

Gerardin DCC, Pereira OCM, Kempinas WG, Florio JC, Moreira EG, Bernardi MM (2005) Sexual behavior, neuroendocrine, and neurochemical aspects in male rats exposed prenatally to stress. Physiol Behav 84:97-104. https://doi.org/10.1016/j.physbeh.2004.10.014

Gouvêa TS, Morimoto HK, de Faria MJSS, Moreira EG, Gerardin DCC (2008) Maternal exposure to the antidepressant fluoxetine impairs sexual motivation in adult male mice. Pharmacol Biochem Behav 90:416-419. https://doi.org/10.1016/j.pbb.2008.03.025

Hanley GE, Brain U, Oberlander TF (2015) Prenatal exposure to serotonin reuptake inhibitor antidepressants and childhood behavior. Pediatr Res 78:174-180. https://doi.org/10.1038/pr.2015.77

Harris SS, MacIag D, Simpson KL, Lin RCS, Paul IA (2012) Dosedependent effects of neonatal SSRI exposure on adult behavior in the rat. Brain Res 1429:52-60. https://doi.org/10.1016/j.brainres. 2011.10.025

Heikkinen T, Ekblad U, Palo P, Laine K (2003) Pharmacokinetics of fluoxetine and norfluoxetine in pregnancy and lactation. Clin Pharmacol Ther 73:330-337. https://doi.org/10.1016/S00099236(02)17634-X

Hernández-Arteaga E, Hernández-González M, Rentería MLR, Almanza-Sepúlveda ML, Guevara MA, Silva MA, Jaime HB (2016) Prenatal stress alters the developmental pattern of behavioral indices of sexual maturation and copulation in male rats. Physiol Behav 163:251-257. https://doi.org/10.1016/j.physbeh.2016.05. 008
Homberg JR, Pattij T, Janssen MCW, Ronken E, De Boer SF, Schoffelmeer ANM et al (2007) Serotonin transporter deficiency in rats improves inhibitory control but not behavioural flexibility. Eur J Neurosci 26:2066-2073. https://doi.org/10.1111/j.1460-9568. 2007.05839.x

Houwing DJ, Buwalda B, van der Zee EA, de Boer SF, Olivier JDA (2017) The serotonin transporter and early life stress: translational perspectives. Front Cell Neurosci 11:1-16. https://doi.org/10.3389/ fncel.2017.00117

Houwing DJ, Ramsteijn AS, Riemersma IW, Olivier JDA (2019a) Maternal separation induces anhedonia in female heterozygous serotonin transporter knockout rats. Behav Brain Res 356:204-207. https://doi.org/10.1016/j.bbr.2018.08.031

Houwing DJ, Staal L, Swart JM, Ramsteijn AS, Wöhr M, de Boer SF, Olivier JDA (2019b) Subjecting dams to early life stress and perinatal fluoxetine treatment differentially alters social behavior in young and adult rat offspring. Front Neurosci 13:1-15. https://doi. org/10.3389/fnins.2019.00229

Kaplan YC, Keskin-Arslan E, Acar S, Sozmen K (2017) Maternal SSRI discontinuation, use, psychiatric disorder and the risk of autism in children: a meta-analysis of cohort studies. Br J Clin Pharmacol 83: 2798-2806. https://doi.org/10.1111/bcp.13382

Kinsley C, Svare B (1986) Prenatal stress reduces intermale aggression in mice. Physiol Behav 36:783-786. https://doi.org/10.1016/00319384(86)90369-0

Kiryanova V, Dyck RH (2014) Increased aggression, improved spatial memory, and reduced anxiety-like behaviour in adult male mice exposed to fluoxetine early in life. Dev Neurosci 36:396-408. https://doi.org/10.1159/000363102

Kiryanova V, Meunier SJ, Vecchiarelli HA, Hill MN, Dyck RH (2016) Effects of maternal stress and perinatal fluoxetine exposure on behavioral outcomes of adult male offspring. Neuroscience 320:281296. https://doi.org/10.1016/j.neuroscience.2016.01.064

Koolhaas JM, Coppens CM, de Boer SF, Buwalda B, Meerlo P, Timmermans PJ a (2013) The resident-intruder paradigm: a standardized test for aggression, violence and social stress. J Vis Exp 1-7. https://doi.org/10.3791/4367

Lisboa SFS, Oliveira PE, Costa LC, Venâncio EJ, Moreira EG (2007) Behavioral evaluation of male and female mice pups exposed to fluoxetine during pregnancy and lactation. Pharmacology 80:49 56. https://doi.org/10.1159/000103097

Maciag D, Simpson KL, Coppinger D, Lu Y, Wang Y, Lin RCS, Paul IA (2006) Neonatal antidepressant exposure has lasting effects on behavior and serotonin circuitry. Neuropsychopharmacology 31:4757. https://doi.org/10.1038/sj.npp.1300823

Masterpasqua F, Chapman RH, Lore RK (1976) The effects of prenatal psychological stress on the sexual behavior and reactivity of male rats. Dev Psychobiol 9:403-411. https://doi.org/10.1002/dev. 420090503

McIlwain KL, Merriweather MY, Yuva-Paylor LA, Paylor R (2001) The use of behavioral test batteries: effects of training history. Physiol Behav 73:705-717. https://doi.org/10.1016/S00319384(01)00528-5

Noorlander CW, Ververs FFT, Nikkels PGJ, van Echteld CJA, Visser GHA, Smidt MP (2008) Modulation of serotonin transporter function during fetal development causes dilated heart cardiomyopathy and lifelong behavioral abnormalities. PLoS One 3:2-11. https://doi. org/10.1371/journal.pone.0002782

Oberlander TF, Reebye P, Misri S, Papsdorf M, Kim J, Grunau RE (2007) Externalizing and attentional behaviors in children of depressed mothers treated with a selective serotonin reuptake inhibitor antidepressant during pregnancy. Arch Pediatr Adolesc Med 161: 22-29. https://doi.org/10.1001/archpedi.161.1.22

Oberlander TF, Papsdorf M, Brain UM, Misri S, Ross C, Grunau RE (2010) Prenatal effects of selective serotonin reuptake inhibitor antidepressants, serotonin transporter promoter genotype (SLC6A4), 
and maternal mood on child behavior at 3 years of age. Arch Pediatr Adolesc Med 164:444-451

Olivier JDA, Vallès A, van Heesch F, Afrasiab-Middelman A, Roelofs JJPM, Jonkers M, Peeters EJ, Korte-Bouws GAH, Dederen JP, Kiliaan AJ, Martens GJ, Schubert D, Homberg JR (2011) Fluoxetine administration to pregnant rats increases anxiety-related behavior in the offspring. Psychopharmacology 217:419-432. https://doi.org/10.1007/s00213-011-2299-z

Patin V, Lordi B, Vincent A, Caston J (2005) Effects of prenatal stress on anxiety and social interactions in adult rats. Dev Brain Res 160:265274. https://doi.org/10.1016/j.devbrainres.2005.09.010

Pawluski JL, Charlier TD, Fillet M, Houbart V, Crispin HT, Steinbusch HW, van den Hove DL (2012a) Chronic fluoxetine treatment and maternal adversity differentially alter neurobehavioral outcomes in the rat dam. Behav Brain Res 228:159-168. https://doi.org/10.1016/ j.bbr.2011.11.043

Pawluski JL, Rayen I, Niessen NA, Kristensen S, van Donkelaar EL, Balthazart J, Steinbusch HW, Charlier TD (2012b) Developmental fluoxetine exposure differentially alters central and peripheral measures of the HPA system in adolescent male and female offspring. Neuroscience 220:131-141. https://doi.org/10.1016/j.neuroscience. 2012.06.034

Pierz KA, Thase ME (2014) A review of vilazodone, serotonin, and major depressive disorder. Prim Care Companion CNS Disord 16: 1-8. https://doi.org/10.4088/pcc.13r01554

Plotsky PM, Meaney MJ (1993) Early, postnatal experience alters hypothalamic corticotropin-releasing factor (CRF) mRNA, median eminence CRF content and stress-induced release in adult rats. Mol Brain Res 18:195-200. https://doi.org/10.1016/0169-328X(93) 90189-V

Rakers F, Rupprecht S, Dreiling M, Bergmeier C, Witte OW, Schwab M (2017) Transfer of maternal psychosocial stress to the fetus. Neurosci Biobehav Rev. https://doi.org/10.1016/j.neubiorev.2017. 02.019

Rayen I, van den Hove DL, Prickaerts J, Steinbusch HW, Pawluski JL (2011) Fluoxetine during development reverses the effects of prenatal stress on depressive-like behavior and hippocampal neurogenesis in adolescence. PLoS One 6. https://doi.org/10.1371/journal.pone. 0024003

Rayen I, Steinbusch HWM, Charlier TD, Pawluski JL (2013) Developmental fluoxetine exposure and prenatal stress alter sexual differentiation of the brain and reproductive behavior in male rat offspring. Psychoneuroendocrinology 38:1618-1629. https://doi. org/10.1016/j.psyneuen.2013.01.007

Rayen I, Steinbusch HWM, Charlier TD, Pawluski JL (2014) Developmental fluoxetine exposure facilitates sexual behavior in female offspring. Psychopharmacology 231:123-133. https://doi. org/10.1007/s00213-013-3215-5

Rhees RW, Fleming DE (1981) Effects of malnutrition, maternal stress, or ACTH injections during pregnancy on sexual behavior of male offspring. Physiol Behav 27:879-882. https://doi.org/10.1016/ 0031-9384(81)90057-3

Rhees RW, Lephart ED, Eliason D (2001) Effects of maternal separation during early postnatal development on male sexual behavior and female reproductive function. Behav Brain Res 123:1-10. https:// doi.org/10.1016/S0166-4328(00)00381-8

Rodriguez-Porcel F, Green D, Khatri N, Harris SS, May WL, Lin RCS, Paul IA (2011) Neonatal exposure of rats to antidepressants affects behavioral reactions to novelty and social interactions in a manner analogous to autistic spectrum disorders. Anat Rec 294:1726-1735. https://doi.org/10.1002/ar.21402
Sandi C, Haller J (2015) Stress and the social brain: behavioural effects and neurobiological mechanisms. Nat Rev Neurosci 16:290-304. https://doi.org/10.1038/nrn3918

Singh Y, Jaiswal AK, Singh M, Bhattacharya SK (1998) Effect of prenatal diazepam, phenobarbital, haloperidol and fluoxetine exposure on foot shock induced aggression in rats. Indian J Exp Biol 36: 1023-1024

Smith JW, Seckl JR, Evans AT, Costall B, Smythe JW (2004) Gestational stress induces post-partum depression-like behaviour and alters maternal care in rats. Psychoneuroendocrinology 29:227-244. https:// doi.org/10.1016/S0306-4530(03)00025-8

Sodhi MSK, Sanders-Bush E (2003) Serotonin and brain development. Int Rev Neurobiol 59:111-174. https://doi.org/10.1016/S00747742(04)59006-2

Stevenson JM (2018) Insights and barriers to clinical use of serotonin transporter pharmacogenetics in antidepressant therapy. Pharmacogenomics 19:167-170. https://doi.org/10.2217/pgs-20170196

Svirsky N, Levy S, Avitsur R (2016) Prenatal exposure to selective serotonin reuptake inhibitors (SSRI) increases aggression and modulates maternal behavior in offspring mice. Dev Psychobiol 58:71-82. https://doi.org/10.1002/dev.21356

Veenema AH, Neumann ID (2009) Maternal separation enhances offensive play-fighting, basal corticosterone and hypothalamic vasopressin mRNA expression in juvenile male rats. Psychoneuroendocrinology 34:463-467. https://doi.org/10.1016/j. psyneuen.2008.10.017

Veenema AH, Blume A, Niederle D, Buwalda B, Neumann ID (2006) Effects of early life stress on adult male aggression and hypothalamic vasopressin and serotonin. Eur J Neurosci 24:1711-1720. https:// doi.org/10.1111/j.1460-9568.2006.05045.x

Veenema AH, Bredewold R, Neumann ID (2007) Opposite effects of maternal separation on intermale and maternal aggression in C57BL/6 mice: link to hypothalamic vasopressin and oxytocin immunoreactivity. Psychoneuroendocrinology 32:437-450. https:// doi.org/10.1016/j.psyneuen.2007.02.008

Vieira ML, Hamada RY, Gonzaga NI, Bacchi AD, Barbieri M, Moreira EG, Mesquita SFP, Gerardin DCC (2013) Could maternal exposure to the antidepressants fluoxetine and St. John's Wort induce longterm reproductive effects on male rats? Reprod Toxicol 35:102-107. https://doi.org/10.1016/j.reprotox.2012.07.006

Wang CT, Shui HA, Huang RL, Tai MY, Peng MT, Tsai YF (2006) Sexual motivation is demasculinized, but not feminized, in prenatally stressed male rats. Neuroscience 138:357-364. https://doi.org/ 10.1016/j.neuroscience.2005.11.026

Weiss IC, Franklin TB, Vizi S, Mansuy IM (2011) Inheritable effect of unpredictable maternal separation on behavioral responses in mice. Front Behav Neurosci 5:1-12. https://doi.org/10.3389/fnbeh.2011. 00003

Yu Q, Teixeira CM, Mahadevia D, Huang Y, Balsam D, Mann JJ, Gingrich JA, Ansorge MS (2014) Dopamine and serotonin signaling during two sensitive developmental periods differentially impact adult aggressive and affective behaviors in mice. Mol Psychiatry 19:688-698. https://doi.org/10.1038/mp.2014.10

Zhou X, Li Y, Ou J, Li Y (2018) Association between maternal antidepressant use during pregnancy and autism spectrum disorder: an updated meta-analysis. Mol Autism 9:1-7. https://doi.org/10.1186/ s13229-018-0207-7

Publisher's note Springer Nature remains neutral with regard to jurisdictional claims in published maps and institutional affiliations. 